Pacific Journal of Mathematics

REDUCIBLE COMPLEMENTS OF LIE ALGEBRA RADICALS 


\title{
REDUCIBLE COMPLEMENTS OF LIE ALGEBRA RADICALS
}

\author{
DAVID J. WiNTER
}

\begin{abstract}
This paper establishes for finite dimensional Lie algebras of characteristic $p$ a counterpart of Levi's "radical splitting theorem" for Lie algebras of characteristic 0 .
\end{abstract}

Reducible Lie algebras are Lie algebras whose solvable radicals Solv $L$ are nilpotent. For every Cartan subalgebra $H$, Solv $L$ has a reducible complement $S$ invariant under ad $H_{s}$, that is, $L=S+\operatorname{Solv} L$ and $S \cap$ $\operatorname{Solv} L$ is nilpotent. The key for choosing $S$ invariant under ad $H_{s}$ is a generalization of Engel's Criteria for Nilpotence. When $L$ is a Lie $p$-algebra, $S$ can be chosen such that every torus of $L$ of maximal rank is in the same toral switching class as some torus of $S$.

1. Introduction. This paper is concerned with the structure of Lie algebras $L$ over a field $k$ of characteristic $p=0$ or $p>0$. Notational conventions follow Jacobsen [2], Seligman [3], Winter [5]. Throughout the paper, all Lie algebras and vector spaces are assumed to be finite dimensional.

In the paper, we prove a variation of Levi's theorem, which is concerned with the existence of complements, in the following sense, of the solvable radical Solv $L$ of $L$. We also indicate briefly in general terms, how our results are used to generate, inductively, structural information about a given Lie algebra.

1.1. Definition. A complement of the solvable radical Solv $L$ of a Lie algebra $L$ is a subalgebra $S$ of $L$ such that $L=S+\operatorname{Solv} L$.

Note that $S / S \cap \operatorname{Solv} L$ is isomorphic to $L / \operatorname{Solv} L$ for any complement $S$ of $\operatorname{Solv} L$ in $L$. Since $L / \operatorname{Solv} L$ is semisimple, we may state this as follows.

1.2. Proposition. $S / \operatorname{Solv} S$ is isomorphic to $L / \operatorname{Solv} L$ for any complement $S$ of $\operatorname{Solv} L$ in $L$.

The "best possible" theorem on existence of complements holds only when the ground field $k$ has characteristic 0 , and counterexamples abound for characteristic $p>0$. 
1.3. THEOREM (Levi [2]). Let L be a Lie algebra over a field of characteristic 0 . Then the solvable radical of $L$ has a semisimple complement $S$.

In this paper, we prove the existence of reducible ad $H_{s}$-stable complements of $\operatorname{Solv} L$ in $L$, given a Cartan subalgebra $H$. We briefly discuss reducible Lie algebras and the use of such reducible complements in $\S \S 2$ and 3. Section 4 is devoted to a generalization of Engel's theorem needed for the proof, in $\$ 5$, of the existence of reducible complements stable under ad $H_{s}$. Questions of unicity, conjugacy and Cartan switching are discussed in $\S 6$, where it is shown that a Lie $p$-algebra $L$ with triangulable radical Solv $L$ has a reducible complement $S$ stable under ad $H_{s}$ such that every torus $T$ of $L$ of maximal dimension can be switched to a torus $T^{\prime}$ which is contained in $S$. Here, ad $H_{s}=\operatorname{span}\left\{(\operatorname{ad} x) p^{i} \mid x \in H, p^{i} \geq\right.$ $\operatorname{dim} L\}$. (Recall from Winter [11] that each element $x$ of a Lie $p$-algebra decomposes as $x=x_{s}+x_{n}$ uniquely where $x_{s}$ and $x_{n}$ are defined in terms of the $p$ th power operation and ad $x=\operatorname{ad} x_{s}+\operatorname{ad} x_{n}$ is decomposition of ad $x$ into its semisimple and nilpotent parts.)

Reducible Lie algebras are characterized in $\$ 2$ in terms of their ad-modules, which we now define. The class of such modules for a given Lie algebra $L$ contains all subquotients of the adjoint (left regular) module $L$ of $L$.

1.4. Definition. An ad-representation for a Lie algebra $L$ is a representation $\rho$ for $L$ such that $\rho(x)$ is nilpotent for all $x \in L$ for which ad $x$ is nilpotent. An ad-module for $L$ is a module for $L$ whose associated representation is an ad-representation.

2. Reducible Lie algebras. When the condition in Theorem 1.3 that $S$ be semisimple is weakened to the condition that $S$ be reducible in the following sense, a revised theorem holds for all Lie algebras $L$ relative to a given Cartan subalgebra $H$.

2.1. Definition. A Lie algebra $S$ is reducible if its solvable radical Solv $S$ is nilpotent.

Letting Nil $S$ denote the (unique) maximal nilpotent ideal of $S$, the nilpotent radical of $S$, it follows that $S$ is reducible if and only if $\operatorname{Solv} S=$ Nil $S$ if and only if $S /$ Nil $S$ is semisimple. This implies that the irreducible ad-modules $V$ for a reducible Lie algebra $S$ coincide with those for $S / \operatorname{Solv} S$, since $(\operatorname{Solv} S) V=\operatorname{Solv}\{0\}$. Taking $V$ to be the adjoint 
module of a Lie algebra $S$ and passing from $V$ to the sum $\bar{V} \sum_{i=1}^{n} \bar{V}_{i}$ of its composition factors $\bar{V}_{i}$, the condition $(\operatorname{Solv} S) \bar{V}_{i}=\{0\}(1 \leq i \leq n) \mathrm{im}$ plies that Nil $S=\operatorname{Solv} S$ and $S$ must be reducible. We state these observations as follows.

2.2. Proposition. A Lie algebra $S$ is reducible if and only if (Solv $S$ ) $V=\{0\}$ for every irreducible ad-module for $S$.

The characterization of reducible Lie algebras stated in Proposition 2.2 is the basis for applications of reducible Lie algebras such as those discussed in $\$ 3$ below.

The following characterization of reducible complements is used in $\S 5$.

2.3. Proposition. $S$ is a reducible complement of the solvable radical of $L$ if and only if:

1. $L=S+\operatorname{Solv} L$;

2. $S \cap \operatorname{Solv} L$ is nilpotent.

3. Application of the existence of reductive complements. We now briefly indicate in general terms how Theorem 5.1 is used to generate, inductively, structural information about a Lie algebra $L$. This method is used in Winter $[9,10]$ to get specific information on root strings in the rootsystem of $L$.

One starts with a subalgebra $B$ of $L$ for which one already has information about the structure of the semisimple algebra $B / \operatorname{Solv} B$ and its irreducible representations. To use this information to obtain information about $L$, one takes a reducible complement $S$ of $\operatorname{Solv} B$ in $B$ and a composition series $L_{1}>L_{2}>\cdots>L_{n}$ of $L$ as $S$-module. One then has:

1. $B / \operatorname{Solv} B$ and $S /$ Nil $S$ are isomorphic;

2. The composition factors $L_{i} / L_{i+1}$ are irreducible modules for $S /$ Nil $S$ when $($ Nil $S) L_{i} \subset L_{i+1}$.

But then such composition factors $L_{i} / L_{i+1}$ can be regarded as irreducible modules for $B / \operatorname{Solv} B$, by way of an isomorphism from $B / \operatorname{Solv} B$ to $S /$ Nil $S$. This enables one to transfer known structural information about the semisimple Lie algebra $B / \operatorname{Solv} B$ and its irreducible representations to $S / \operatorname{Solv} S$ and the composition factors $L_{i} / L_{i+1}$ which are ad-modules for $S$.

An important feature of the procedure outlined above is that structural information about a semisimple Lie algebra $B / \operatorname{Solv} B=\bar{B}$ which is expressed relative to a specified Cartan subalgebra $\bar{H}$ is transferred to a 
reducible complement $S$ provided that $S$ be stable under ad $H_{s}$. Such a choice of $S$ is always possible, by Theorem 5.1.

4. A generalization of Engel's Theorem. Engel's theorem states that a Lie algebra $L$ is nilpotent if ad $L$ consists of linear transformations. A linear version of it states that a Lie subalgebra $L$ of the algebra $\mathrm{Hom}_{k} V$ of linear transformations of a vector space $V$ is nilpotent if it consists of linear transformations. The purpose of this section is to generalize these two theorems as a prelude to $\$ 6$. The generalization is based on the theorem of Jacobson [1] on weakly closed sets of nilpotent linear transformations, and on methods of ascent and descent.

We begin by proving a linear version of parts 1 and 2 of the following Engel's theorem for graded Lie algebras over a group $A$.

4.1. TheOREM (Winter [8]). Let $L=\sum_{a \in A} L_{a}$ be an A-graded Lie algebra. Suppose that ad $L_{1}$ consists of nilpotent linear transformations. Then:

1. if $A$ is torsion free, $L$ is nilpotent;

2. if $p>0, A$ is a p-group and $(\operatorname{ad} L)^{p} \subset \operatorname{ad} L, L$ is nilpotent;

3. if $A$ is cyclic, $L$ is solvable.

In the above theorem, an A-graded Lie algebra is a Lie algebra $L$ which is the direct sum $L=\sum_{a \in A} \oplus L_{a}$ of subspaces $L_{a}$ (some of which may be $\{0\})$ such that $\left[L_{a}, L_{b}\right] \subset L_{a b}$ for all $a, b \in A$. In the following theorem, an $A$-graded linear Lie algebra is an $A$-graded Lie algebra $L=\sum_{a \in A} L_{a}$ which is a Lie subalgebra of $\operatorname{Hom}_{k} V$ where $V$ is a finite dimensional graded vector space $V=\sum_{a \in A} \oplus V_{a}$ such that $L_{a} V_{b} \subset V_{a b}$ for all $a, b \in A$.

4.2. Theorem. Let $L=\sum_{a \in A} L_{a}$ be a graded linear Lie algebra, $V=\sum_{a \in A} V_{a}$ the underlying graded vector space. Suppose that $L_{1}$ consists of nilpotent transformations of $V$. Then:

1. if either $A$ is torsionfree, or $p>0, A$ is a (finite or infinite) p-group and $L^{p} \subset L$, then $L$ consists of nilpotent linear transformations and $L$ is nilpotent:

2. if $A$ is cyclic, $L$ is solvable;

3. if $A$ is cyclic and $V_{0}=\{0\}, L$ consists of nilpotent linear transformations and $L$ is nilpotent.

Proof. By the theorem of Jacobson [2] on weakly closed sets of nilpotent linear transformations, it suffices to show that $x$ is nilpotent for all $x \in L_{a}, a \in A$. For $a=1$, this is true by hypothesis. Let $a \neq 1$ and 
note that $x^{n} V_{b} \subset V_{a} n_{b}$ for all $b \in A$ and all positive integers $n$. For $A$ torsionfree or $V_{0}=\{0\}$, this implies that $x$ is nilpotent. Suppose next that $p>0$, and $A$ is a $p$-group. Since $L$ is finite dimensional, we can assume that $A$ is finite without any loss of generality. Let $|a|=p^{e}$ and $L^{p} \subset L$. Then $x^{p^{e}} V_{b} \subset V_{a^{p^{e}} b_{b}}=V_{b}$ for all $b \in A$. Writing $x^{p^{e}} \in L^{p^{e}} \subset L$ as $x^{p^{e}}=$ $y \in L$, and writing $y$ as $y=\sum_{c \in A} y_{c} \in \sum_{c \in A} L_{c}$, we conclude for $v_{b} \in V_{b}$ that $V_{b}$ contains $y v_{b}=\sum_{c \in A} y_{c} v_{b}$. Thus, $y_{c} V_{b}=0$ for $c \neq 1, b \in A$. It follows that $y=y_{1} \in L_{1}$ and $y$ is nilpotent. Since $y=x^{p^{e}}, x$ is nilpotent, as asserted. Finally, suppose that $A$ is cyclic. Since $L_{0}$ consists of nilpotent linear transformations, so does ad $L_{0}$, so that $L$ is solvable by Theorem 4.1.

We now formulate the theorem needed for $\$ 5$ in terms of the $p$-closure of $L$. This is defined as the span $\bar{L}$ of $\left\{x^{p^{e}} \mid x \in L, e=\right.$ $0,1,2, \ldots\}\left(p^{e}=1\right.$ if $\left.p=0\right)$, so that $\bar{L}=L$ for $p=0$. The growth in passing from $L$ to $\bar{L}$ is related to the strength of Theorem 4.3 for a given $L$, which is greatest when $L=\bar{L}$. The departure $\bar{L} / L$ of $\bar{L}$ from $L$ is constrained by the following relationships between $L$ and $\bar{L}$, observed in Winter $[6,7]$, which show that $\bar{L} / L$ is an abelian Lie algebra isomorphic to $\hat{H} /(\hat{H} \cap H)$ :

1. $\bar{L}^{(1)} \subset L$ and $\bar{L}=\hat{H}+L$ for every Cartan subalgebra $\hat{H}$ of $\bar{L}$;

2. $\bar{L}$ has a Cartan subalgebra $\hat{H}$ such that $\hat{H} \cap L \subset H$. Those relationships are not needed for the proof of Theorem 4.3, but they and the construction in Winter [7] of $\hat{H}$ from $H$ are relevant to understanding when it can be applied. For the proof, one does need to know that $[\bar{N}, \bar{L}] \subset[N, L]$ for any subalgebra $N$ of $\operatorname{Hom}_{k} V$ such that $[N, L] \subset L$. To see this, observe, firstly, that $[\bar{N}, L] \subset L$ since the normalizer of $L$ is closed under $p$ th powers. Then note that $x \in \bar{N}, y \in L$ implies $\left[x, y^{p^{e}}\right]$ $\in L$, which is clear upon rewriting $\left[x, y^{p^{e}}\right]$ as $[\ldots[x, y], y, \ldots, y](y$ occurs $p^{e}$ times) with $[x, y] \in[\bar{N}, L] \subset L$ and $y \in L$.

4.3. TheOREM. Let $L$ be a Lie subalgebra of $\operatorname{Hom}_{k} V$ and let $N$ be a nilpotent Lie subalgebra of $\mathrm{Hom}_{k} V$ such that $[N, L] \subset L$. Suppose that $\bar{L}_{0}(\operatorname{ad} N)$ consists of nilpotent linear transformations. Then $L$ consists of nilpotent linear transformations and $L$ is nilpotent.

Proof. We take $K$ to be the algebraic closure of $k$ and ascend from $k, V, N, L, \bar{L}$ to $K, V_{K}=K \otimes_{k} V, N_{K}=K \otimes_{k} N, L_{K}=K \otimes_{k} L, \bar{L}_{K}=$ $K \oplus_{k} \bar{L}$. Since $\bar{L}_{0}(\operatorname{ad} N)$ is the solution space of a system of linear equations (defined by $p^{e}$ th powers $(\operatorname{ad} x)^{p^{e}}$ with $p^{e} \geq \operatorname{dim} \bar{L}$ of ad $x \in$ $\operatorname{ad} N$, for $p>0$, and by semisimple parts of $\operatorname{ad} x \in \operatorname{ad} N$ for $p=0$ ), 
$\bar{L}_{K 0}\left(\operatorname{ad} N_{K}\right)$ is the $K$-span $K \bar{L}_{0}(\operatorname{ad} N)$ of $\bar{L}_{0}(\operatorname{ad} N)$. Thus, the zero subalgebra of the graded Lie algebra $\bar{L}_{K}=\sum_{a \in G} \bar{L}_{K a}\left(\operatorname{ad} N_{K}\right)=\sum_{a \in G} \bar{L}_{K a}$ is $\bar{L}_{K 0}=K \bar{L}_{0}(\operatorname{ad} N)$. It follows from Engel's theorem that $\bar{L}_{0}(\operatorname{ad} N)$ and, therefore, $K \bar{L}_{0}(\operatorname{ad} N)=\bar{L}_{K 0}$ are triangulable algebras of nilpotent linear transformations of $V_{K}$. Now $\bar{L}_{K}$ is a graded linear Lie algebra with underlying graded vector space $V_{K}=\sum_{a \in G} V_{K a}\left(N_{K}\right)$. Since $G$ is a finitely generated additive group of functions from $N_{K}$ to $K, G$ is torsionfree for $p=0$ or an elementary abelian $p$-group for $p>0$. For $p>0$, the condition $L^{p} \subset L$ implies that $\left(L_{K}\right)^{p} \subset L_{K}$, by Theorem 11, p. 190 of Jacobson [1]. We may, therefore, conclude that $\bar{L}_{K}$ consists of nilpotent transformations of $V_{K}$ and $\bar{L}_{K}$ is nilpotent, by Theorem 4.2. Thus, $L$ is a nilpotent Lie algebra of nilpotent linear transformations of $V$, as asserted.

The above counterpart of Engel's theorem for linear Lie algebras has, as immediate consequence, the following counterpart of Engel's theorem for abstract Lie algebras.

4.4. TheOrem. Let $L$ be a Lie algebra and let $N$ be a nilpotent Lie algebra of derivations of $L$. Suppose that $(\overline{\operatorname{ad} L})_{0}(\operatorname{ad} N)$ consists of nilpotent linear transformations. Then $L$ is nilpotent.

5. Existence of reducible complements relative to a given Cartan subalgebra. In Theorem 5.1 below, we show that Lie algebra $L$ with Cartan subalgebra $H$ has a reducible complement $S$ stable under ad $H_{s}$, that is, ad $H_{s}(S) \subset S$ and:

1. $L=S+\operatorname{Solv} L$

2. $S \cap \operatorname{Solv} L$ is nilpotent.

We then show for $L / \operatorname{Solv} L$ simple that the proper ideals of any minimal ad $H_{s}$-stable reducible complement $S$ of $\operatorname{Solv} L$ in $L$ are contained in Nil $S$.

5.1. THEOREM. Let $L$ be a Lie algebra over a field $k$, and let $H$ be a Cartan subalgebra of $L$. Then the solvable radical of $L$ has a reducible ad $H_{s}$-stable complement $S$. If $L$ is restricted, $S$ can be taken to be restricted.

Proof. If Solv $L$ is nilpotent, it suffices to let $S=L$.

Suppose next that $R=\operatorname{Solv} L$, is not nilpotent. By Theorem 4.3 $(\overline{\operatorname{ad} R})_{0}(\operatorname{ad} \operatorname{ad} H)$ has an element $X_{1}$ which is not nilpotent on $R$. Since we need the action of $X_{1}$ on $L$ later in the proof, ad $x(x$ in $R$ or $L)$ is 
regarded as left translation on $L$, rather than on $R$. Let $R_{1}=R_{0}\left(X_{1}\right)=$ $\left\{x \in R \mid X_{1}^{\operatorname{dim} L}(x)=0\right\}$ and note that $R \neq R_{1}$. We continue to choose $X_{2}, X_{3}, \ldots$ and $R_{2}, R_{3}, \ldots$ according to the rules.

1. $X_{i+1} \in\left(\overline{\operatorname{ad} R_{i}}\right)_{0}\left(\operatorname{ad} H_{s}\right)$ where $X_{i+1}$ is not nilpotent on $R_{i}$;

2. $R_{i+1}=R_{i 0}\left(X_{i+1}\right)$.

Note that $R_{i}$ is stable under ad $H$ since $\left[X_{i}^{p^{e}}\right.$, ad $\left.H\right]=0$ for $p^{e} \geq \operatorname{dim} L$. Since $R_{1}>R_{2}>\cdots>R_{i}$, by condition (1), there is a terminal $R_{i}$ which is reached when the condition " $X$ is nilpotent on $R_{i}$ for all $X \in$ $\left(\overline{\operatorname{ad} R_{i}}\right)_{0}(\operatorname{ad} H)$ " is met. But this is the condition of Theorem 4.3 for nilpotency of ad $R_{i}$, so that $R_{i}$ is nilpotent. Since $X_{1} \in(\overline{\operatorname{ad} R})_{0}(\operatorname{ad} H)$, we have $H \subset L_{1}=L_{0}\left(X_{1}\right)=\left\{x \in L \mid X_{1}^{p^{e}}(x)=0\right\}\left(p^{e} \geq \operatorname{dim} L\right)$. Moreover, $X_{1} \in \overline{\operatorname{ad} R}$ and ad $R(L) \subset R$ imply that $X_{1}(L) \subset R$, so that $L_{1}+$ $R=L_{0}\left(X_{1}\right)+R=L_{0}\left(X_{1}\right)+L_{*}\left(X_{1}\right)=L$. Since $X_{2} \in\left(\overline{\operatorname{ad} R_{1}}\right)_{0}(\operatorname{ad} H)$, we have $H \subset L_{2}=L_{10}\left(X_{2}\right)$. Moreover, we have $X_{2}(L) \subset R$ and $L_{2}+R$ $=L_{10}\left(X_{2}\right)+R=L_{10}\left(X_{2}\right)+L_{1 *}\left(X_{2}\right)+R=L_{1}+R=L$. Continuing, we have $H \subset L_{i}$ and $L=L_{i}+R$. Finally, we have $L_{i} \cap R=L_{0}\left(X_{1}\right) \cap$ $L_{0}\left(X_{2}\right) \cap \cdots \cap L_{0}\left(X_{i}\right) \cap R=R_{0}\left(X_{1}\right) \cap R_{0}\left(X_{2}\right) \cap \cdots \cap R_{0}\left(X_{i}\right)=R_{i}$, so that $L_{i} \cap R$ is nilpotent. The following conditions are now established, which completes the proof of the existence of $S$ since we may take $s=L_{i}$ :

1. $L=L_{i}+\operatorname{Solv} L, H \subset L_{i}$;

2. $L_{i} \cap \operatorname{Solv} L$ is nilpotent.

Clearly, if $L$ is restricted, $S=L_{i}$ is also.

By the above theorem, a Lie algebra $L$ with Cartan subalgebra $H$ has a reducible complement stable under ad $H_{s}$; so $L$ has a minimal ad $H_{s}$-stable complement $S$. The ideals $I$ of $S$ correspond to ideals $I+\operatorname{Solv} L$ of $L$, since $L=S+\operatorname{Solv} L$. The ideals $S^{i}, S^{(i)}$ are ad $H$-stable ideals of $S$ If $L / \operatorname{Solv} L$ is simple (and nonzero), then $L=S^{2}+\operatorname{Solv} L$ and $S^{2}$ is a reducible complement. But then $S=S^{2}$ by the minimality. More generally, we have the following consequence of Theorem 5.2.

5.2. TheOREM. Let $L$ be a Lie algebra with Cartan subalgebra $H$ and let $S$ be a minimal ad $H_{s}$-stable reducible complement of $\operatorname{Rad} L$ in $L$. Suppose that $L / \operatorname{Solv} L$ is simple. Then $S$ is a local Lie algebra in the sense that every proper ad $H_{s}$-stable ideal of $S$ is contained in Nil $S$.

The condition that $S$ be a local Lie algebra is generally much stronger than the condition that $S / \mathrm{Nil} S$ be simple. In fact, this is illustrated by such easily constructed algebras as $S=T \oplus U$ (direct) with $U$ nonzero and nilpotent, $T /$ Nil $T$ simple (and nonzero), Nil $T$ nonzero, which are not local since $T$ is a proper ideal not contained in $\operatorname{Nil} S=\operatorname{Nil} T \oplus U$. 
Proof of Theorem 5.2. Let $I$ be a proper ideal of $S$. Since $S$ is an ideal of $H+S, I^{\infty}$ is an ideal of $H+S$ by Jacobson [2], Exercise 9, p. 29. Since $I^{\infty}$ is ad $H$-stable, the ideal $I^{\infty}+\operatorname{Solv} L$ of $L$ is proper, by minimality of $S$. By simplicity of $L / \operatorname{Solv} L$, it follows that $I^{\infty} \subset \operatorname{Solv} L$, so that $I^{\infty} \subset S \cap \operatorname{Solv} L=\operatorname{Nil} S$. But then the nilpotent ideal $I+$ Nil $S /$ Nil $S$ of the simple Lie algebra $S /$ Nil $S$ must be 0 and $I \subset$ Nil $S$.

6. Uniqueness, conjugacy and switching of Cartan subalgebras. For Lie algebras $L$ over an algebraically closed field of characteristic 0 , the rich supply of automorphisms exp ad $x=\sum_{n=0}^{\infty}(\operatorname{ad} x)^{n} / n !(\operatorname{ad} x$ nilpotent $)$ insure that the group Aut $L$ of automorphisms of $L$ act transitively on the set of Cartan subalgebras, and on the set of semisimple complements (Levi factors), of $L$.

For Lie algebras $L$ over an algebraically closed field of characteristic $p>0$, the objects of discussion for this section, such exp ad $x$ can be defined by truncating to $\exp \operatorname{ad} x=\sum_{n=0}^{p-1}(\operatorname{ad} x)^{n} / n$ !. However, the truncated exp ad $x$ are not always automorphisms, and Cartan subalgebras are not always conjugate under Aut $L$.

The effect of this is that nonconjugate Cartan subalgebras must be taken into account. The emerging classification theory for simple Lie algebras of Block-Wilson [1], Wilson [4] involves starting from a given Cartan subalgebra $H$, and then using the exponential Cartan switching operators $e^{x}$ of Winter [11] to change $H$ to a Cartan subalgebra $H^{\prime}=$ $e^{x_{1}} \cdots e^{x_{n}} H$ in an "optimal" conjugacy class. Theorem 6.1 shows that, under certain circumstances, there is no loss of generality in doing this within a given reducible complement $S$ containing $H$.

There is no known general procedure for "switching" nonnilpotent subalgebras, such as reducible complements of $S$, thereby generating switching classes of such $S$. However, Theorem 6.1 can be viewed as a method for comparing reducible complements $S, S^{\prime}$ of a Lie $p$-algebra $L$ which respectively contain tori $T, T^{\prime}$ of $l$ of maximal dimension.

In the theorem, a Lie $p$-algebra $L$ is p-triangulable if its restricted representations are triangulable or, equivalently, if $x^{p^{e}}=0$ for $p^{e} \geq \operatorname{dim} L$ for all $x \in L^{(1)}$.

6.1. TheOREM. Let $L$ be a Lie p-algebra with maximal torus $H$ over an algebraically closed field of characteristic $p>0$ such that $\operatorname{Solv} L$ is $p$-triangulable. Then $L$ has a reducible complement $S$ of $\operatorname{Solv} L$ containing $H$ such that every torus $T$ of $L$ of maximal dimension can be switched to a torus $T^{\prime}=e^{x_{t}} \cdots e^{x_{n}} T$ contained in $S$. 
Proof. By the special properties of Lie $p$-algebras and their Cartan subalgebras discussed in Winter [7], $S=L_{0}(\operatorname{ad}(H \cap \operatorname{Solv} L))$ has intersection $S \cap \operatorname{Solv} L=(\operatorname{Solv} L)_{0}(\operatorname{ad}(H \cap \operatorname{Solv})) \supset \hat{H}$, a maximal torus of $\operatorname{Solv} L$. Since $\operatorname{Solv} L$ is $p$-triangulable, it follows that $\operatorname{Solv} L=\hat{H}+$ $(\operatorname{Solv} L)^{(1)}$ where $(\operatorname{Solv} L)^{(1)}$ is a $p$-nil ideal of $L$. Now $L=S+(\operatorname{Solv} L)^{(1)}$ with $S \cap \operatorname{Solv} L$ nilpotent, so that $S$ is a reducible complement. The decomposition $L=S+(\operatorname{Solv} L)^{(1)}$ of $L$ with $(\operatorname{Solv} L)^{(1)}$ a $p$-nil ideal of $L$ enables us to invoke Theorem 4.3 of Winter [11] in concluding that $S$ contains some $e^{x_{1}} \cdots e^{x_{n}} T$.

\section{REFERENCES}

[1] Richard Block and Robert Lee Wilson, The simple Lie p-algebra of rank 2, Annals of Math., 115 (1982), 93-168.

[2] Nathan Jacobson, Lie Algebras, Interscience, New York, 1962.

[3] George Seligman, Modular Lie Algebras, Ergebnisse der Math. und ihrer Grenzgebiete, Band 40, Springer-Verlag, New York, 1967.

[4] Robert Lee Wilson, Simple Lie algebras of total rank one, Trans. Amer. Math. Soc. 236 (1978), 287-295.

[5] David J. Winter, Abstract Lie Algebras, MIT Press, Cambridge, Mass., 1972.

[6] , Cartan decompositions and Engel subalgebra triangulability, J. Algebra, 62, No. 2, (1980), 400-417.

[7] Cartan subalgebras of a Lie algebra and its ideals II, Pacific J. Math., 92, No. 1, (1981), 14-15.

[8] , On groups of automorphisms of Lie algebras, J. Algebra, 8, No. 2, (1968), 131-142.

[9] _ Root systems of simple Lie algebras, J. Algebra, 97, No. 1, (19xx), 166-180.

[10] __ Symmetric Lie algebras, J. Algebra, 97, No. 1, (19xx), 130-165.

[11] _ On the toral structures of Lie p-algebras, Acta Math., 123 (1968), 69-81.

Received March 31, 1983

UNIVERSITY OF MICHIGAN

ANN ARBOR, MI 48109 



\section{PACIFIC JOURNAL OF MATHEMATICS EDITORS}

\author{
V. S. VARADARAJAN \\ (Managing Editor) \\ University of California \\ Los Angeles, CA 90024 \\ HERBERT Clemens \\ University of Utah \\ Salt Lake City, UT 84112 \\ R. FINN \\ Stanford University \\ Stanford, CA 94305
}

HERMANN FLASCHKA

University of Arizona

Tucson, AZ 85721

RAMESH A. GANGOLLI

University of Washington

Seattle, WA 98195

VAughan F. R. JONES

University of California

Berkeley, CA 94720

ROBION KIRBY

University of California

Berkeley, CA 94720
C. C. MoOrE

University of California

Berkeley, CA 94720

H. SAMELSON

Stanford University

Stanford, CA 94305

HAROLD STARK

University of California, San Diego

La Jolla, CA 92093

\section{ASSOCIATE EDITORS}
R. AREnS
E. F. BECKENBACH
B. H. NEUMANN
F. WOLF
K. YOSHIDA (1906-1982)

\section{SUPPORTING INSTITUTIONS}

UNIVERSITY OF ARIZONA

UNIVERSITY OF BRITISH COLUMBIA

CALIFORNIA INSTITUTE OF TECHNOLOGY

UNIVERSITY OF CALIFORNIA

MONTANA STATE UNIVERSITY

UNIVERSITY OF NEVADA, RENO

NEW MEXICO STATE UNIVERSITY

OREGON STATE UNIVERSITY
UNIVERSITY OF OREGON UNIVERSITY OF SOUTHERN CALIFORNIA STANFORD UNIVERSITY UNIVERSITY OF HAWAII UNIVERSITY OF TOKYO UNIVERSITY OF UTAH WASHINGTON STATE UNIVERSITY UNIVERSITY OF WASHINGTON 


\section{Pacific Journal of Mathematics}

\section{Vol. 128, No. $1 \quad$ March, 1987}

Anthony Peter Bahri and Peter Gilkey, The eta invariant, $\operatorname{Pin}^{c}$ bordism, and equivariant $\operatorname{Spin}^{c}$ bordism for cyclic 2-groups $\ldots \ldots \ldots \ldots \ldots \ldots 1$

Friedrich-Wilhelm Bauer, Extensions of generalized homology theories . . . 25

Marilyn Breen, A characterization theorem for compact unions of two

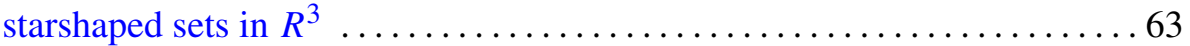

Boniface Ihemotuonye Eke, Special generating sets of purely inseparable extension fields of unbounded exponent $\ldots \ldots \ldots \ldots \ldots \ldots \ldots \ldots \ldots \ldots$

Robert William Gilmer, Jr. and William James Heinzer, Jónsson $\omega_{0}$-generated algebraic field extensions $\ldots \ldots \ldots \ldots \ldots \ldots \ldots \ldots \ldots . . .61$

Guido Lupacciolu, Holomorphic continuation in several complex variables

Douglas C. McMahon, Jaap C. S. P. van der Woude and Ta-Sun Wu,

Connectedness related to almost periodicity of compositions of flow

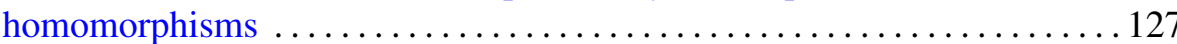

Sophocles Mercourakis, Some characterizations of analytic metric spaces

Peter Frederick Stiller, The Picard numbers of elliptic surfaces with many

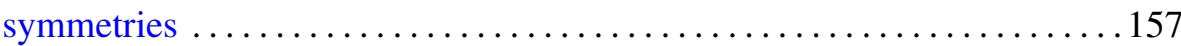

David J. Winter, Reducible complements of Lie algebra radicals ........ 191 Rade Živaljević, On a cohomology theory based on hyperfinite sums of microsimplexes 